\title{
SEGMENTATION AND RECOGNITION OF CONNECTED HANDWRITTEN CHARACTERS
}

\author{
Shehan Panditharatne and Lochandaka Ranathunga ${ }^{1}$ \\ Faculty of Information Technology, University of Moratuwa, Sri Lanka \\ ${ }^{1} \mathrm{Dr}$.
}

\begin{abstract}
Optical Character Recognition is still an open problem in computer science. This paper explores segmentation of connected (touching/overlapping) handwritten characters through implicit segmentation and explicit segmentation. Explicit segmentation is achieved through rules based on background thinning and analyzing the contour hierarchy. Implicit segmentation is achieved through generating a dataset of connected characters and training a CNN. This research was inspired by the need to recognize math documents containing algebraic equations. Recognition of 72 characters (alphanumeric and math symbols) are achieved through the proposed methods. The scope of the implemented OCR is limited to maximum of two connected characters.
\end{abstract}

\section{KEYWORDS}

Implicit, Explicit Segmentation, Connected, Touching Characters

\section{INTRODUCTION}

Handwritten text is still a widely used method of communication in today's world. Even with the advancements in digital text applications, handwritten text is still the medium for text in fields such as education. Thus, it's vital to make advancements in recognizing such texts. There exist many researches regarding handwritten text recognition. Even though many advancements have been made, handwritten character recognition is still considered an open problem.

This research paper was based on our final year thesis on recognizing handwritten documents containing algebraic equations. Given sufficient space between lines of the document, they can be easily segmented by smearing the text into blobs using morphological operations. In the segmented line, characters separated by spaces can be segmented through the peaks and throughs of the vertical projection. This leaves us with single characters or connected (touching/overlapping) characters. This paper focuses on recognition of these single or connected characters using implicit and explicit segmentation methods since the previous steps are of common knowledge. The scope of this research however is limited to two connected (touching/overlapping) characters. The source code and the sample images used for evaluating the system can be found at: (Panditharatne, 2020).

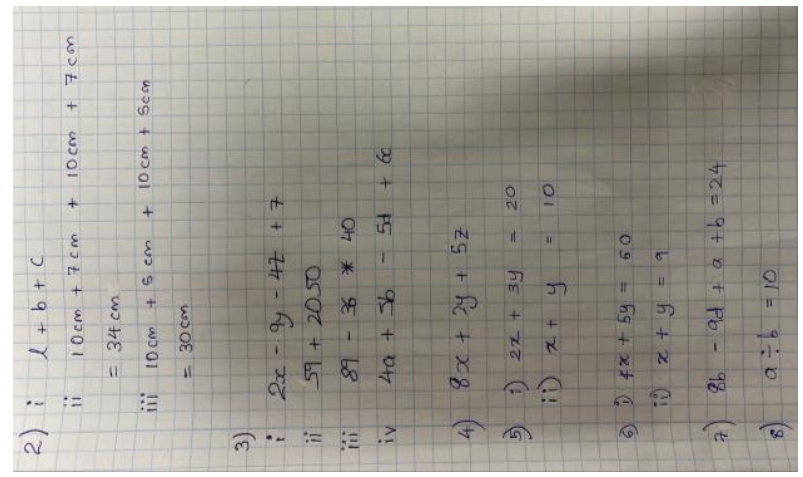

Figure 1. Sample document 


\section{RELATED WORK}

Character segmentation is considered one of the most crucial steps in handwritten OCR (KAUR, BAGHLA, \& KUMAR, 2015). Correct segmentation of characters is imperative for correct classification of characters in later stages.

Although characters that are separated by spaces can be easily segmented there are challenges in segmenting touched or overlapping characters. Touching, connected, overlapping, intersecting characters will all be referred as connected characters from here onwards for simplicity.

In research, character segmentation is broadly classified into two main techniques (Rehman, Mohamad, \& Sulong, 2009).

\subsection{Explicit Segmentation}

A classical approach where an image of a word sequence is partitioned to several, individual character images using rules.

In (Casey \& Eric, 1996) Casey et al. demonstrates a method of using vertical projection (vertical histogram) of a line to find separation of characters. Vertical projection is the count of foreground pixels in each column of the line. The minimums(valleys) of the projection serves as a detector for white space between successive letters thus the segmentation points. However, when touching or overlapping characters are introduced this method fails to correctly segment the characters. Thus, this method serves as a successful method for segmentation non-cursive handwriting.

In (Lu, Chi, Siu, \& Shi, 1999) Lu et al. proposes an approach of segmenting hand-written two-digit strings by thinning the background of the image. After retrieving the skeleton of the background, the algorithm locates several feature points that can be used to segment the image. Possible segmentation paths are then constructed between the detected feature points of the upper background contour and the lower background contour. Since there can be multiple possible segmentation paths, they are ranked using fuzzy rules generated from a decision-tree approach. Then the segmentation paths with the highest ranks are tested according to an acceptance criterion.

\subsection{Implicit Segmentation}

Recognition based segmentation method where character recognition and segmentation are achieved simultaneously. Segmentation can be regarded as a byproduct of the recognition process (Rehman, Mohamad , \& Sulong, 2009). Thus, the recognizer decides on the best segmentation hypothesis. In (Zhan, Lyu, \& Lu, 2018) Zhan et al. proposes a new architecture based on a CNN to recognize handwritten digit strings. A Connectionist Temporal Classification (CTC) layer is used in the output layer to calculate the loss and decode the feature sequence.

In (Meany \& Arola, 2018) Meany et al. trains a CNN on the IAM Handwriting word dataset. They have limited the length of the words to 1-6 characters to achieve best performance. They have trained the dataset on a 1-layer CNN and a 3-layer CNN. They have achieved accuracies of $71.6 \%$ and $76.9 \%$ respectively for the test set.

\subsection{Character Classification}

In (Meany \& Arola, 2018), Meany et al. trains a CNN and a ResNet-50 on the EMNIST dataset to achieve character classification. They have trained the model on the EMNIST balanced dataset where there are 47 classes. The CNN reaches an accuracy of $84.8 \%$ and the ResNet reaches an accuracy of $88.5 \%$ on the test set.

\section{METHODOLOGY}

The methods proposed for implicit segmentation and explicit segmentation are discussed below. It's important to note that single character images or connected character images (segmented from vertical projection) are the inputs into the proposed methods. Connected characters are limited to two connected characters. 


\subsection{Implicit Segmentation}

In implicit segmentation, character segmentation and character recognition are achieved simultaneously. This can be achieved using a Convolutional Neural Network. A dataset containing single characters and two connected characters will have to be generated as none such datasets were available online.

\subsubsection{Generation of the Dataset}

The dataset will be generated using merged EMNIST (Cohen, Afshar, Tapson, \& Schaik, 2017) dataset (for 52 characters and 10 numbers) and the CROHME dataset (for 10 mathematical symbols). The CROHME dataset is a dataset of Online handwritten characters. However, through offline_CROHME (Huynh, 2018) tool it's possible to convert the online handwritten characters to offline character images. Only 10 mathematical symbols are chosen from the CROHME dataset as they are the most basic math symbols. The symbols are $-(),,+,-, /,=,[$,$] , division, *$. The whole merged dataset is converted to binary colors because the pre-processing module of the OCR turns all images to binary colors using OTSU thresholding and the mathematical symbols of the CROHME dataset is in binary color. Thus, the grayscale color depth of EMNIST is sacrificed.

Images are generated by randomly taking a character from the merged dataset (EMNIST + CROHME) and combining it with a another randomly taken character from the merged dataset. The merged dataset should be balanced such that each character class (out of 72) has the equal number of images. Thus, will allow us to generate a dataset that is more balanced, as characters are taken from the merged dataset at random for combining.

Characters are combined by placing the second character where the first character ends. The characters are combined such that some images are touching characters and some images are overlapping characters. This variation between overlapping and touching characters can be achieved by randomly shifting left (by around 3 pixels) where the second character is placed.

Each generated image will have two labels. First label for the first character of the image and the second label for the second character in the image. For single character images the first label will contain the character in the image and the second label will contain a number to represent a blank character (72). The merged dataset has 72 (EMNIST+CROHME) types of characters and thus the generated dataset has 5256 (72x73) possible character combinations/classes. Each combination will have at least 600 images thus in total there are around 3153600 images (600x5256). The exact number of images in the generated dataset is 3230 980. Each image in the dataset is sized at 28x56 (height $\mathrm{x}$ width) and is in binary colors. The following figure shows some samples of the generated images and their labels.

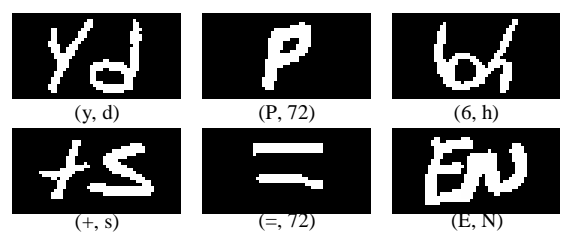

Figure 2. Samples of the generated dataset

\subsubsection{Training the Model}

The generated dataset will be trained on a 4-layer CNN, 6-layer CNN and on ResNet-50. The problem can be addressed as a multi label classification or a multi class classification. If the problem is addressed as a multi label classification, the output of the NN will have $145(72+73)$ outputs and each image will have two labels (ex: $-0,8$ or 0,72$)$. If the problem is addressed as a multi class classification, the $\mathrm{NN}$ will have $5256(72 \times 73)$ outputs. Each class will denote one combination between two characters.

When training the model, it was observed that it's easier to address the problem as a multi class classification as the training images were too large to fit on memory and thus had to be loaded from the hard drive. Therefore, Keras flow from directory method was used to load the images from the hard drive.

For training the ResNet, images were resized to 56x112 and then was transformed into RGB. Train test split of 90/10 was used to train and evaluate the model. 


\subsection{Explicit Segmentation}

First, a classification must be performed to recognize weather the image contains a single character or connected characters. The following approaches were implemented and tested.

- Through hierarchical clustering - First, the width and the white pixel count of each image is calculated. Through hierarchical clustering of these values, it is possible to classify characters as single characters and connected characters. The cluster of the image with the largest white pixel count will be chosen as the connected character cluster. The other cluster will be the single character cluster.

- Through a CNN - This classification can be addressed as a binary classification problem where a single character is classified as 0 and connected characters classified as 1 . Since there are no such public datasets available for this purpose, a dataset was generated. This dataset was generated in similar fashion to the dataset generated in the implicit segmentation section. Instead of 5256 possible classes used in the implicit segmentation dataset, this dataset has two (binary) classes. The dataset contains 318672 images where $50 \%$ of the images are single images and the rest are connected character images.

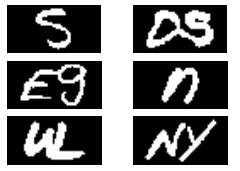

Figure 3. Samples of the generated dataset

A CNN with 8 Convolutional Layers will be used to perform the classification.

If the image is recognized as a single character, it can be directly sent to the character classifier to be classified. If the image is of connected characters, it will have to be further processed to segment into two characters.

\subsubsection{Background Thinning Based Segmentation}

The first step in segmenting the image is to perform background thinning of the input image. The following images are of input images and their respective background thinned images.

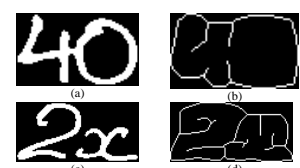

Figure 4. (a,c)Connected Character Image (b,d)Background thinned images

The next step is to separate overlapping characters from touching characters. This can be achieved by checking the contours of the background image. If there are two separable contours in the image, the image is recognized as two overlapping characters (d image above). Thus, they can be easily segmented by creating masks of the contours. Then the single character images are sent to the character classifier to be classified.

If the image doesn't contain two separable contours, it's taken as a touching character image (b image above). In such images, the background thinned image is divided into 3 sections along the $\mathrm{x}$ axis (width wise) and the middle section is chosen for further processing (red area in the c image below). It's assumed that, the candidate segmentation line between the characters is situated in this middle section. A kernel is applied to find candidate points in the upper part of the background skeleton (bounded by red in d,e image below). Another kernel is applied to find candidate points in the lower part of the background skeleton (bounded by green in d,e image below). These points are then connected to form a segmentation line (f image below). 

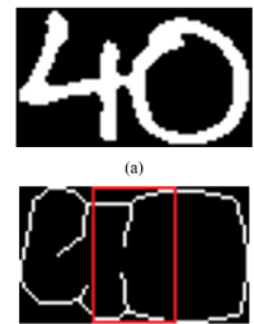

(c)

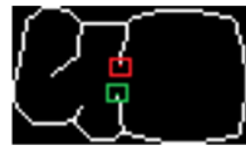

(e)

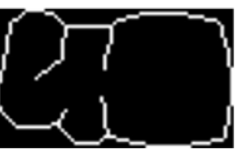

(b)

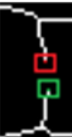

(d)

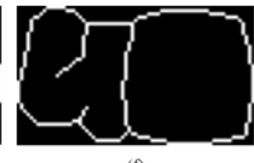

Figure 5. (a)Touching character image (b)Background thinned image (c)The middle section outlined in red (d) Candidate points found (Red- candidate point in upper part, Green- Candidate point in lower part) (e)Candidate points in the background image (f)Final image with connected candidate points

The above figure demonstrates how an input image (a) is processed to find the candidate points that are to be connected. Which in turn gives the segmentation line upon connecting the points (f).

In order to find the candidate point, in the upper part of the background thinned image, the following convolution kernel can be used.

\begin{tabular}{|c|c|c|}
\hline 2 & 2 & 2 \\
\hline-10 & 10 & -10 \\
\hline-10 & -10 & -10 \\
\hline
\end{tabular}

Figure 6. Convolution kernel to find candidate points in the upper part

Checking this convolution against a threshold of $3060(255 \times 10+255 \times 2)$ allows us to find following types of candidate points.
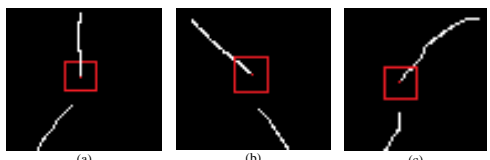

Figure 7. Types of candidate points found by the kernel

In order to find the candidate point in the lower part of the background thinned image, the above kernel can be applied after being rotated by $180^{\circ}$.
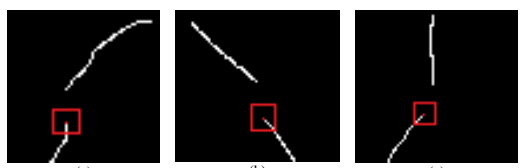

Figure 8 . Types of candidate points found by the rotated kernel

If no candidate points can be found, then the image is not segmentable by the current algorithm.

The most trivial case is when there is only one candidate point each per upper and lower part of the skeleton is found. They can be directly connected to form the segmentation line. However, a choice must be made in case there are more than one point in each upper and lower parts of the skeleton. Points ( $\mathrm{x}, \mathrm{y})$ found on the upper part of the skeleton will be referred to as top points and points (x,y) found on the lower part of the skeleton will be referred to as bottom points from here onwards.

If there are no top points and there is at least one bottom point, the bottom point's $\mathrm{X}$ value is chosen as the top point's $\mathrm{X}$ value and 0 will be chosen as the $\mathrm{Y}$ value.

If there are is least one top point and no bottom points, the top point's $\mathrm{X}$ value is chosen as the bottom points $\mathrm{X}$ value and the last row of the image is chosen as the bottom points $\mathrm{Y}$ value. In case there are multiple top points, the points are sorted such that the points whose $\mathrm{Y}$ values are the largest (closest to the bottom of the image) are at the start of the array and the first point is chosen as the top point. 
If there are multiple top points and only one bottom point, the distances between the bottom point and the top points are calculated. The top point that gives the lowest distance to the bottom point is chosen as the top point.

Through connecting the candidate top point and the candidate bottom point, a segmentation line can be drawn and thus the image can be segmented to two characters using the contour hierarchy method.

\subsubsection{Splitting Characters using Contour Hierarchy}

If the background skeleton is separated clearly as follows (figure b, d), contour hierarchy can be used to split the image into two characters.

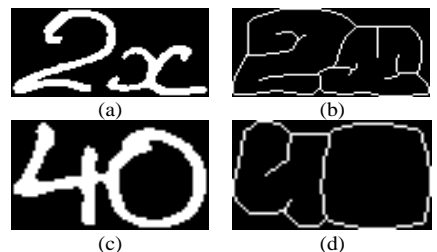

Figure 9. Images that can be split using contour hierarchy

The first step of the process is to find the contour hierarchies of the background skeleton as a tree. This retrieves the contours and creates a full family hierarchy list. The contour that encircles the whole region of interest is the contour at the top of the hierarchy (parent) and it will be assigned the value 0 . The following figure shows the contour hierarchy where $(a, e)$ images are of the background skeleton, $(b, f)$ images are of the parent contour (rank 0) and (c, d, g, h) images are the child contours of the parent (rank 0) contour.

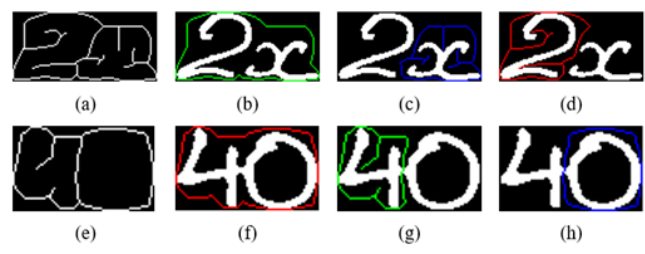

Figure 10. Contour hierarchy

If there are two child contours under the rank 0 parent contour, then the image can be segmented to two-character images. In case of overlapping characters (b), the image is segmented along the space between the characters. In case of touching characters (f), the background skeleton should be sent with the candidate segmentation line (image e).

If two child contours under the rank 0 contour can't be found, then this algorithm marks the image as being not segmentable.

\subsection{Character Classification}

In this module single characters are classified to their respective labels. The inputs are the single characters in the document or the segmented characters. The dataset will be a combination of the EMNIST dataset and the CROHME dataset with a total of 72 characters. These 72 characters are made up of the characters of the alphabet (A-Z and a-Z), numbers (0-9) and mathematical symbols ((, ), +, -, /, =, [, ], division, *.). The CROHME dataset is a dataset of Online handwritten characters. However, through offline_CROHME [10] tool it's possible to convert the online handwritten characters to offline character images. The whole dataset will be pre-processed into binary colors because the pre-processing module of the OCR turns the document to binary colors using OTSU thresholding and the offline CROHME dataset is available only in binary color. The dataset will be trained on 4-layer CNN, 6-layer CNN and on ResNet50. The merged dataset has 880709 training images and 140862 test images. 


\section{EVALUATION}

\subsection{Module Wise Evaluation}

\subsubsection{Connected Character Recognition (Binary)}

This is a sub module in the explicit segmentation module. This classifier is used to recognize weather an image is of a single character or connected characters (two connected characters). This module is tested on the dataset that is generated. There are 31868 test images in the dataset.

Hierarchical clustering failed to correctly cluster the data in this dataset. Many irrelevant points were being recognized as clusters. Therefore, $\mathrm{K}$-Means clustering with $\mathrm{K}=2$ was used to cluster the data instead.

Table 1. Single or multiple character classification (binary)

\begin{tabular}{|l|l|}
\hline & Test Accuracy \\
\hline 8-layer CNN & $98.57 \%$ \\
\hline K-Means clustering (K=2) & $92.07 \%$ \\
\hline
\end{tabular}

\subsubsection{Single Character Classifier}

This is a sub module in the explicit segmentation module. Segmented and single characters are classified using this classifier. The test set has 140862 (EMNIST+ offline CROHME) images belonging to 72 classes.

Table 2. Single character classifier accuracy

\begin{tabular}{|l|l|}
\hline Model & Test Accuracy \\
\hline 4-layer CNN & $87.73 \%$ \\
\hline 6-layer CNN & $88.11 \%$ \\
\hline ResNet-50 & $88.01 \%$ \\
\hline
\end{tabular}

\subsubsection{Single or Connected Character Classification}

Here the images of single characters or images of connected characters are classified. The segmentation and classification of characters are achieved simultaneously in the implicit segmentation module.

For explicit segmentation, 8-layer CNN was used to recognize connected characters followed by background thinning based segmentation. Finally, the segmented single characters are recognized using the single character 6-layer CNN model.

This evaluation is performed using the generated dataset mentioned in Implicit Segmentation section. Out of 3230980 images in the dataset 323098 images are of the test set.

Table 3. Single or connected character classification

\begin{tabular}{|l|l|l|}
\hline \multicolumn{2}{|c|}{ Test Accuracy } \\
\hline \multirow{2}{*}{ Implicit segmentation } & 4-layer CNN & $63.85 \%$ \\
\cline { 2 - 3 } & 6-layer CNN & $63.77 \%$ \\
\cline { 2 - 3 } & ResNet-50 & $96.01 \%$ \\
\hline Explicit segmentation & $31.61 \%$ \\
\hline
\end{tabular}

\subsection{Evaluation on Real Data}

The OCR as a whole was tested on 321 handwritten characters. These characters are spread over 4 documents containing characters, numbers and math symbols. These documents can be found at (Panditharatne, 2020). There are 215 single characters and 53 connected character pairs in the test set.

For testing implicit segmentation, ResNet-50 model was used.

For explicit segmentation, an 8-layer CNN was used to recognize connected characters followed by background thinning based segmentation. Finally, the segmented characters are recognized using the single character 6-layer CNN.

The documents were also tested with Google's Tesseract OCR (v5.0.0-alpha.20190623 with Leptonica). The backgrounds of the documents were removed through OTSU thresholding to improve accuracy.

Table 4. Accuracy on real data

\begin{tabular}{|l|c|c|c|}
\hline & Accuracy on all characters & Single character Accuracy & $\begin{array}{l}\text { Touch/overlapping character } \\
\text { accuracy }\end{array}$ \\
\hline $\begin{array}{l}\text { Implicit Segmentation } \\
\text { ResNet-50) }\end{array}$ & $190 / 321=59.19 \%$ & $119 / 215=55.81 \%$ & $70 / 106=66.03 \%$ \\
\hline Explicit segmentation & $188 / 321=58.56 \%$ & $143 / 215=66.51 \%$ & $45 / 106=42.45 \%$ \\
\hline Google Tesseract OCR & $160 / 321=49.84 \%$ & $121 / 215=56.27 \%$ & $39 / 106=36.79 \%$ \\
\hline
\end{tabular}




\section{CONCLUSION}

Looking at the evaluation accuracy for real data, it can be observed that the implicit segmentation module is less accurate on single characters. This is likely due to each character class in the generated dataset containing only around 600 images. Thus, adding all the single character images from EMNIST and offline CROHME to the generated dataset should improve the single character accuracy. However, this will make the dataset highly imbalanced. Therefore, more research should be done in this aspect.

The explicit segmentation module and the implicit segmentation module performs similarly on real documents. Although the implicit segmentation module has high accuracy on connected characters it performs a bit poorly on single characters compared to the explicit segmentation module.

The implicit segmentation module performs well on connected characters compared to the explicit segmentation model as expected. Convolutional Neural Networks perform better in image recognition problems compared to explicit rule-based methods. However, more accuracy can be gained through cleaning and expanding the dataset.

The proposed background thinning based segmentation algorithm fails to correctly segment characters in some instances. One instance is when the correct segmentation points lay outside of the middle part of the image. Another instance is when the convolution kernels fails to find any candidate points or finds the wrong candidate points. The following figure shows where segmentation line is drawn using incorrectly detected candidate points.

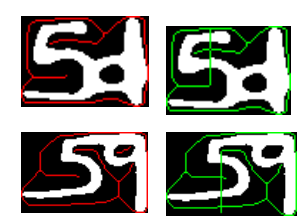

Figure 11. Incorrectly segmented images

The evaluation also shows that the proposed methods performs better on the tested documents than Google's Tesseract OCR. 2020).

The source code and sample images used for evaluating the system can be found at: (Panditharatne,

\section{REFERENCES}

Casey, G. R., \& Eric, L. (1996). A Survey of Methods and Strategies in Character Segmentation. IEEE TRANSACTIONS ON PATTERN ANALYSIS AND MACHINE INTELLIGENCE, 18.

Cohen, G., Afshar, S., Tapson, J., \& Schaik, A. (2017). EMNIST: an extension of MNIST to handwritten letters. The MARCS Institute for Brain, Behaviour and Development Western Sydney University Penrith, Australia.

Huynh, D. (2018, December 14). Converting CROHME dataset for Online-handwritting recognition to Offline-handwritting recognition. Retrieved September 8, 2019, from https://github.com/vndee/offline-crohme

KAUR, A., BAGHLA, S., \& KUMAR, S. (2015). STUDY OF VARIOUS CHARACTER SEGMENTATION TECHNIQUES. International Journal of Advances in Science Engineering and Technology, 3(3).

Lu, Z., Chi, Z., Siu, W.-C., \& Shi, P. (1999). A background-thinning-based approach for separating and recognizing connected handwritten digit strings. Pattern Recognition, 32, 921-933.

Meany, C., \& Arola, M. (2018). Optical Character Recognition via Deep Learning. CA: Deep Learning, Stanford University.

Panditharatne, S. (2020, April 9). xyz-ocr test images. (GitHub) Retrieved April 9, 2020, from GitHub: https://github.com/shehan360/xyz-ocr/tree/master/TestImages

Rehman, A., Mohamad , D., \& Sulong , G. (2009). Implicit Vs Explicit based Script Segmentation and Recognition: A Performance Comparison on Benchmark Database. Int. J. Open Problems Compt. Math, 2.

Zhan, H., Lyu, S., \& Lu, Y. (2018). Handwritten Digit String Recognition using Convolutional Neural Network. International Conference on Pattern Recognition. Beijing. 